\title{
XXI. The effect of ionization of air on electrical oscillations, and its bearing on long-distance wireless telegraphy
}

\section{Prof. G.W.O. Howe}

To cite this article: Prof. G.W.O. Howe (1914) XXI. The effect of ionization of air on electrical oscillations, and its bearing on long-distance wireless telegraphy, Philosophical Magazine Series 6, 27:157, 213-215, DOI: 10.1080/14786440108635079

To link to this article: http://dx.doi.org/10.1080/14786440108635079

曲 Published online: 08 Apr 2009.

Submit your article to this journal $₫$

Џll Article views: 4

Q View related articles $₫$ 
This method again may be applied to the beam with end loading, thus ambne (fig. $b$ ) is the primary deflexion diagram for a beam of two spans under a uniform load, in which the centre span has sunk by an amount $\delta$. Find the characteristic points $\mathrm{S}_{2}, \mathrm{~T}_{2}$, at each side of the support $b$, carefully bearing in mind that area $a \mathrm{kbm}$ is the one to be used in the expression for the distance of the characteristic point from the base-line ac. Next modify these characteristic points in accordance with the formulæe already considered in reference to continuous beains under lateral loading only.

Conclusion.-It will be seen that the method of successive graphical approximation is applicable to beams under endlong and lateral loading either continuous or discontinuous. The end load may or may not pass through the centrode of the beam section. For large columns under practical conditions it is usually unnecessary to take into account the moments due to lateral deflexion, but under special circumstances of slender columns these moments may become of importance. For a simple system of loading on a beam of one span only the analytical method of solution presents many advantages, but if the lateral loading is complicated by the presence of unsymmetrical concentrated loads or by distributed loads of varying intensity, the graphical method is shorter and easily applied. For an analysis of a beam or tie continuous over several supports and subjected to endlong loadings it is, although long and complicated, the only method available.

XXI. The Effect of Ionization of Air on Electrical Oscillations, and its bearing on Long-Distance Wireless Telegraphy*. By Prof. G. W. O. Howe, Imperial College, South Kensington $t$.

WHILE it is true that Dr. Eccles' theory of the refraction of electromagnetic waves in the upper atmosphere is based on the assumption that ionization malses the atmosphere conducting, it must not be overlooked that this conductivity must, as Dr. Eecles has shown, be of a different type to that of ordinary materials, if it is to account for the observed phenomena. When a charged ion of molecular mass is situited in an alternating electric field

* See Paper with this title by Prof. Barton and Mr. Kilby, Phil. Mag. Oet. 1913, p. 567 .

$\dagger$ Communicated by the Author. 
214 Effect of Ionization of Air on Electrical Oscillations.

it is subjected to an alternating force, which causes it to oscillate about a mean position. If it is entirely free from frictional restraint, the acceleration in any direction will be a maximum when the force and therefore the field is a maximum in that direction. The molecule must have its maximum displacement in the opposite direction at this moment, and the electric current due to its motion will therefore lag $90^{\circ}$ behind the electric tield. This current is therefore in exact opposition to the pure displacement current, and has the effect of reducing the apparent dielectric constant of the medium. The velocity of propagation of electromagnetic waves should therefore be increased.

If the molecular motion is not free from frictional restraint, the current will not be in exact quadrature but will have a power-component, the relative magnitudes of the two components being dependent, among other things, on the freedom of molecular motion, that is, on the vacuum.

This whenomenon must not be confused with the comparatively well-known peculiarities of leaky condensers, $i$. e., condensers shunted with a non-inductive resistance. Here the leakage current is in phase with the electric force, and therefore in quadrature with the displacement current. If $\mathrm{R}, \mathrm{L}, \mathrm{C}$, and $\frac{1}{\mathrm{~S}}$ represent the resistance, inductance, capacity, and shunt resistance of an oscillatory circuit, we have

$$
f=\frac{1}{2 \pi} \sqrt{\frac{1}{\mathrm{LC}}-\left(\frac{\mathrm{R}}{2 \mathrm{~L}}-\frac{\mathrm{S}}{2 \mathrm{C}}\right)^{2}} .
$$

As the leakance $\mathrm{S}$ is increased from zero it counteracts the effect of $R$ in decreasing the natural frequency, until, when $S=\frac{R O}{L}$, the frequency reaches a maximum. Further increase in $\mathbb{S}$ causes the frequency to decrease. This has no connexion, however, with the type of conductivity considered above.

Ordinary leakage cannot increase the relocity of propagation of electromagnetic waves.

This is readily seen from the formula for the velocity of propagation along a telephone line, which is

where $\quad \omega=2 \pi f$

$$
v=\frac{\omega}{q}
$$

and

$$
2 q^{2}=\sqrt{\left(\mathrm{R}^{2}+\omega^{2} \mathrm{~L}^{2}\right)\left(\mathrm{S}^{2}+\omega^{2} \mathrm{C}^{2}\right)}-\mathrm{RS}+\omega^{2} \mathrm{CL},
$$


where the symbols refer to the values for unit length of the line. If $\mathrm{R}=0$, this reduces to

$$
\frac{2}{v^{2}}=\operatorname{LC}\left(\sqrt{1+\frac{\mathrm{S}^{2}}{\omega^{2} \mathrm{C}^{2}}}+1\right)
$$

from which it is seen that any increase in the leakage $S$ causes a decrease in the velocity.

Now the results published by Prof. Barton and Mr. Kilhy show agreement with the ordinary theory of the leaky condenser, and therefore indicate that the condenser with a dielectric of ionized air at atmospheric pressure had that type of conductivity or leakage which cannot cause an increase in the velocity of propagation, but can only cause a decrease of velocity and a certain amount of dissipative absorption.

The statement that " the work yields some experimental support to the theory of Dr. Eccles" appears therefore to be unjustified.

It would appear necessary to repeat the experiments with various vacua in the vessel containing the air condenser before the results could have any bearing on the theory put forward by Dr. Eccles.

XXII. The Existence of Uranium $Y$. By Frederick Soddr, M.A., F.R.S.*

[Plate II.]

I N. ANTONOFF (Phil. Mag. 1913 [6] xxvi. p. 1058) (T. states that he has been able to repeat his experiments, which led him two years ago to conclude that a new product, uranium $\mathrm{Y}$, of half-period 1.5 days, existed in the uranium series, with some purified uranyl nitrate obtained from me, and with which A. Fleck in this laboratory (Phil. Mag. 1913 [6] xxv. p. 710) was unable to obtain the same effects. I have pleasure in being able to confirm Antonoff's main resuit of an abnormal decay of the soft $\beta$-rays of uranium $\mathrm{X}$, also with some of the same material. I approached the subject from a point of view which it seemed might account for the discrepancy between the two experimenters. It "ppeared possible that Antonoff's uranium $Y$ might be isotopic $\dagger$ with aranium $\mathrm{X}$, and that any chemical operation

* Communicated by the Author.

† The terms " isotopic elements" or "isotopes" are convenient to indicate elements occupying the same place in the periodic table and therefore chemically indistinguishable and non-separable. See 'Nature,' December 4th, 1913, p. 400. 